\title{
Review
}

\section{Wide-field and two-photon imaging of brain activity with voltage- and calcium-sensitive dyes}

\author{
Ryota Homma ${ }^{1, *}$, Bradley J. Baker ${ }^{1}$, Lei Jin ${ }^{1}$, Olga Garaschuk ${ }^{2}$, \\ Arthur Konnerth ${ }^{3}$, Lawrence B. Cohen ${ }^{1,4}$ and Dejan Zecevic ${ }^{1}$ \\ ${ }^{1}$ Department of Cellular and Molecular Physiology, Yale University School of Medicine, \\ 333 Cedar Street, New Haven, CT 06520, USA \\ ${ }^{2}$ Institute of Physiology Department II, Eberhard Karls Universität Tübingen, 72076 Tübingen, Germany \\ ${ }^{3}$ Institute of Neuroscience, Technical University Munich, 80802 Munich, Germany \\ ${ }^{4}$ RedShirtImaging, LLC, Decatur, GA 30030, USA
}

This review presents three examples of using voltage- or calcium-sensitive dyes to image the activity of the brain. Our aim is to discuss the advantages and disadvantages of each method with particular reference to its application to the study of the brainstem. Two of the examples use wide-field (onephoton) imaging; the third uses two-photon scanning microscopy. Because the measurements have limited signal-to-noise ratio, the paper also discusses the methodological aspects that are critical for optimizing the signal.

The three examples are the following. (i) An intracellularly injected voltage-sensitive dye was used to monitor membrane potential in the dendrites of neurons in in vitro preparations. These experiments were directed at understanding how individual neurons convert complex synaptic inputs into the output spike train. (ii) An extracellular, bath application of a voltage-sensitive dye was used to monitor population signals from different parts of the dorsal brainstem. We describe recordings made during respiratory activity. The population signals indicated four different regions with distinct activity correlated with inspiration. (iii) Calcium-sensitive dyes can be used to label many individual cells in the mammalian brain. This approach, combined with two-photon microscopy, made it possible to follow the spike activity in an in vitro brainstem preparation during fictive respiratory rhythms.

The organic voltage- and ion-sensitive dyes used today indiscriminatively stain all of the cell types in the preparation. A major effort is underway to develop fluorescent protein sensors of activity for selectively staining individual cell types.

Keywords: two-photon microscopy; one-photon (wide-field) microscopy; voltage-sensitive dyes; calcium-sensitive dyes; neural networks

\section{INTRODUCTION}

An optical measurement of brain activity by the use of a molecular probe can provide invaluable information in a variety of circumstances. The obvious advantage of any imaging method is the possibility of simultaneous measurements from many locations. This is especially important in the study of the nervous system where many parts of an individual cell, or many cells, or many interconnected regions, are simultaneously active. In addition, optical recording offers the possibility of recording from neuronal processes that are too small or fragile for electrode recording.

Optical methods for measuring activity can have high spatial resolution. In one of the examples presented in the following, the resolution is of the order of a few microns; in another example, it is easy to

*Author for correspondence (ryota.homma@yale.edu).

One contribution of 17 to a Discussion Meeting Issue 'Brainstem neural networks vital for life'. distinguish the activity of individual cells. However, in vivo optical recordings are limited to areas of the brain that are visible, and in these areas, limited to a recording depth of approximately $0.4 \mathrm{~mm}$ using twophoton microscopy and approximately $100 \mu \mathrm{m}$ for resolving dendrites using wide-field microscopy. Light scattering by the brain limits both the penetration of the excitation light and the recovery of the emitted fluorescent photons.

Using a molecular probe has advantages (and disadvantages) over monitoring intrinsic signals. For optical imaging of membrane potential, the use of molecular probes has resulted in greatly increased signal size in comparison with potential-dependent intrinsic signals such as light scattering and birefringence (Cohen et al. 1968). In addition, with the introduction of protein activity sensors, there is now the possibility for cell-type-specific staining. Furthermore, many of the molecular probes are fast, with a time resolution in microseconds or milliseconds but others, especially the protein-based biosensors, are substantially slower. 


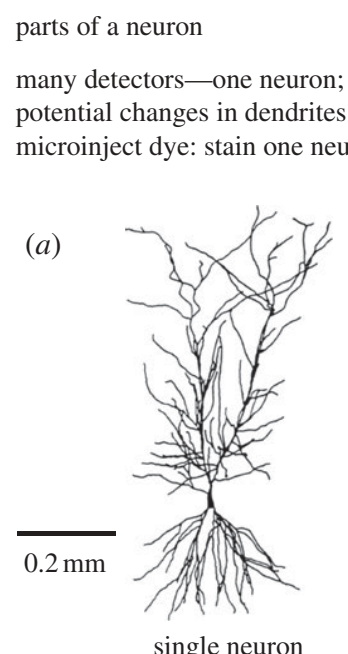

single neuron

\section{individual neurons}

one detector-one neuron; follow spike activity of many individual neurons; bath-applied dye

(b)

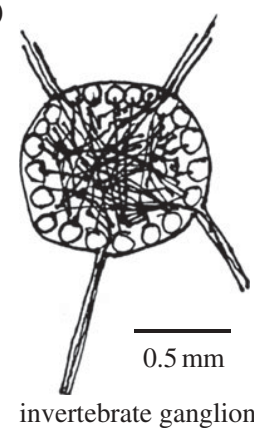

population signals

one detector-many neurons; signals are population average; vertebrate brain;

bath-applied dye, transported dye

(c)

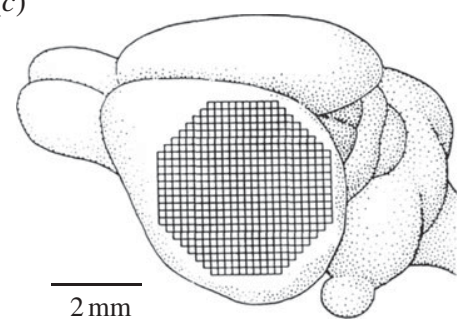

Figure 1. Schematic drawings of the three kinds of measurements described as examples in the text. (a) An individual cortical hippocampal CA1 pyramidal cell. Each pixel of an $80 \times 80$ pixel charge-coupled device (CCD) camera would receive light from a small part of the dendrite, axon or cell body of the neuron. An optical measurement of membrane potential would provide important information about how the neuron converts its synaptic input into its spike output. (b) A drawing of a slice through an invertebrate ganglion with its cell bodies in a cortex around the outside and neuropil in the middle. Here each detector would receive light from one or a small number of cell bodies. A voltage-sensitive dye measurement of spike activity while the nervous system is generating a behaviour would provide important information about how the behaviour is generated. (c) A vertebrate brain with a superimposed pixel array. Each pixel of the array would receive light from thousands of cells and processes. The signal would be the population average of the change in membrane potential in those cells and processes.

In addition, there are probes that are sensitive to various ions and metabolic intermediates. With these advantages comes the responsibility for demonstrating that possible pharmacological and photodynamic effects caused by these probes are minimal (see the following).

Figure 1 illustrates three qualitatively different applications in neurobiology where imaging has been useful. In order to know how a neuron integrates its synaptic input into its action potential output, one needs to be able to measure membrane potential everywhere that synaptic inputs arrive and at the places where spikes are initiated (figure 1a). In order to understand how a nervous system generates a behaviour, it is important to measure the action potential activity of many participating neurons (figure $1 b$ ). Responses to sensory stimuli and generation of motor output in the vertebrate brain are often accompanied by activation of many neurons in widespread brain areas (figure 1c); optical imaging allows measurement of population signals from multiple areas simultaneously. In these three instances, optical recordings have provided information about the function of the nervous system that was previously unobtainable.

\section{SIGNAL SOURCES}

\section{(a) Voltage-sensitive dyes}

Several different optical properties of membrane-bound organic dyes are sensitive to membrane potential including fluorescence, absorption, dichroism, birefringence, fluorescence resonance energy transfer (FRET), second harmonic generation and resonance Raman absorption (Teisseyre et al. 2007). Because many applications in neurobiology have involved fluorescence measurements, these will be emphasized.

The voltage-sensitive dye signals described in this paper are 'fast' signals (Cohen \& Salzberg 1978) that arise from membrane-bound dye; they follow changes in membrane potential with a time course that is rapid compared with the rise time of an action potential. Figure $2 a$ illustrates the kind of result that is used to define a voltage-sensitive dye. In the giant axon from a squid, these optical signals are fast, following membrane potential with a time constant of $<10 \mu \mathrm{s}$ (Loew et al. 1985) and their size is linearly related to the size of the change in potential (e.g. Gupta et al. 1981). Because the signals are linearly related to membrane potential, they report both depolarizations and hyperpolarizations. These organic dyes provide a direct, fast and linear measure of the change in membrane potential of the stained membranes.

Several voltage-sensitive dyes have been used to monitor changes in membrane potential in a variety of preparations. Figure $2 b$ illustrates four different chromophores (the merocyanine dye, XVII, was used for the measurement illustrated in figure $2 a$ ). For each chromophore, approximately 100 analogues have been synthesized in an attempt to optimize the signal-to-noise ratio that can be obtained in a variety of preparations. (This screening was made possible by synthetic efforts of three laboratories: J. Wang, R. Gupta and A. Waggoner then at Amherst College; R. Hildesheim and A. Grinvald at the Weizmann Institute; and J. Wuskell and L. Loew at the University of Connecticut Health Center.) For each of the four chromophores illustrated in figure $2 b$, there were 10 or 20 dyes that gave approximately the same signal size on squid axons (Gupta et al. 1981). However, dyes that had nearly identical signal size on squid axons could have very different responses in other preparations, and thus tens of dyes usually have to be tested in an effort to obtain the largest possible signal.

The following rules of thumb seem to be useful. First, each of the chromophores is available with a 


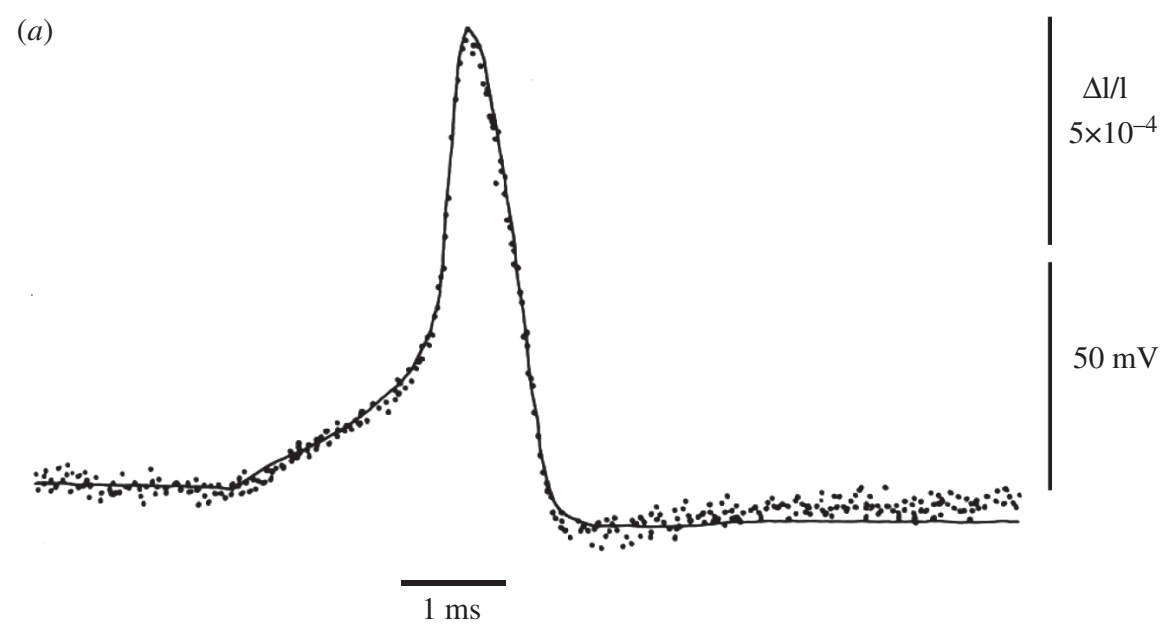

(b)

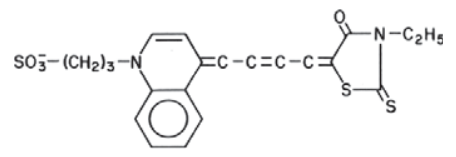

XVII, merocyanine, absorption, birefringence

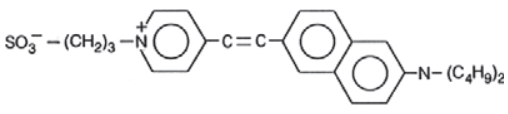

di-4-ANEPPS, styryl, fluorescence

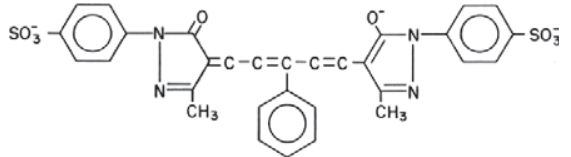

RH155, oxonol, absorption

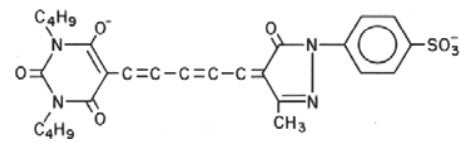

XXV, oxonol, fluorescence, absorption

Figure 2. (a) Changes in transmitted light intensity (absorption; dots) of a squid giant axon stained with a merocyanine dye, XVII, during a membrane action potential (smooth trace) recorded simultaneously with an intracellular electrode. The change in absorption and the action potential had the same time course. The response time constant of the light-measuring system was $35 \mu \mathrm{s} ; 32$ sweeps were averaged. (b) Organic voltage-sensitive dyes: examples of four different chromophores that have been used to monitor membrane potential. The oxonol dye, RH155, and its analogue RH482 (not shown), are commercially available as NK3041 and NK3630 from Hayashibara Biochemical Laboratories Inc./Kankoh-Shikiso Kenkyusho Co. Ltd., Okayama, Japan. The oxonol, XXV (WW781) and styryl, di-4-ANEPPS, are available commercially as dye R-1114 and D-1199 from Invitrogen-Molecular Probes, Junction City, OR. Merocyanine dyes, including XVII (WW375, NK2495), and its analogue, NK2761, need negotiation with Hayashibara Biochemical Laboratories. (Modified from Ross et al. 1974.)

fixed charge that is either a quatenary nitrogen (positive) or a sulphonate (negative). Generally, the positive dyes have given larger signals in vertebrate preparations. Second, each chromophore is available with carbon chains of several lengths. The more hydrophilic dyes (methyl or ethyl) work best if the dye has to penetrate through a compact tissue (vertebrate brain) or needs to travel a long distance in a dendritic tree.

Studies have been made to determine the molecular mechanisms that give rise to potential-dependent optical properties. There is evidence for three different mechanisms (for different dyes). These are dipole rotation, electrochromism and a potential-sensitive monomer-dimer equilibrium (Waggoner \& Grinvald 1977; Loew et al. 1985; Fromherz et al. 1991).

\section{(i) Pharmacological and photodynamic effects}

In several experimental preparations, it was shown that pharmacological and photodynamic effects were small at the dye concentrations and light intensities that were used (e.g. Cohen \& Lesher 1986; Grinvald et al. 1988; Nakashima et al. 1992; Momose-Sato et al. 1995; Zecevic 1996; Palmer \& Stuart 2006; Canepari et al. 2007).

\section{(b) Calcium-sensitive dyes}

Figure $3 b$ shows the chemical structure of a calciumsensitive dye, Calcium Green-1, together with a plot of its fluorescence spectrum as a function of the free calcium concentration (figure $3 a$ ). This dye signal reaches 50 per cent of its maximum at a calcium concentration of approximately $0.2 \mu \mathrm{M}$. In contrast to the voltage-sensitive dyes, the calcium dyes are located intracellularly. The dye is presumed to be in the cytoplasm and to report changes in the calcium concentration in the cytoplasm, although some of the dye may be in intracellular compartments. Calcium dyes are generally slower to respond to abrupt changes in calcium concentration in comparison with voltagesensitive dye responses to abrupt changes in membrane potential. Figure $4 a$ compares the time course of voltage and calcium signals in an in vitro turtle olfactory bulb following stimulation of the olfactory nerve. The signals were measured from the same location on the preparation. The calcium signal reaches its peak more slowly and the signal returns to the baseline much more slowly. In part, the slower return is because of the time taken for the cell to reduce the free calcium concentration back to the resting level. 


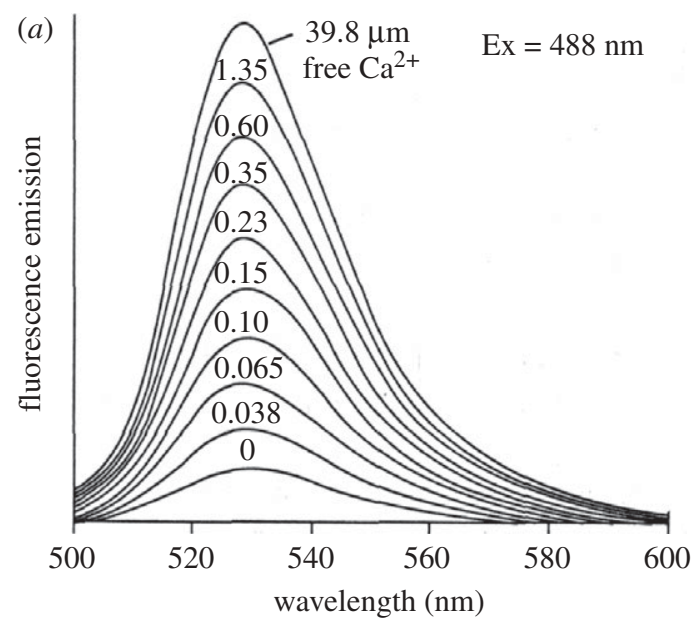

(b)

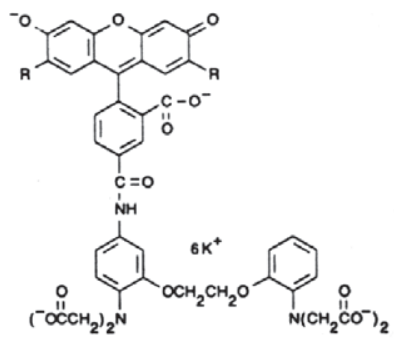

Figure 3. The Calcium Green-1 dextran emission spectrum as a function of calcium concentration (a) and its chemical structure $(b)$. Data taken from the Handbook of Fluorescent Probes and Research Chemicals, sixth edition, Invitrogen-Molecular Probes.

In addition, because the calcium dyes also act as buffers of calcium, the calcium signal in the presence of the dye may substantially outlast the change in calcium concentration that would occur in the absence of the dye (Neher \& Augustine 1992; Helmchen et al. 1996).

\section{(c) Intrinsic signals}

A third optical signal of brain activity that has been used extensively is the intrinsic signal (Blasdel \& Salama 1986; Grinvald et al. 1986). These signals occur in the absence of added dyes. They are thought to reflect mainly the changes in blood flow and haemoglobin oxygenation that occur in response to changes in brain activity. Figure $4 b$ shows a comparison of the calcium signal and the intrinsic signal from a glomerulus in the mouse olfactory bulb (Wachowiak \& Cohen 2003). The intrinsic signals do not reach a peak until approximately $5 \mathrm{~s}$ after the start of the odorant stimulus; they are substantially slower than the calcium signal. Intrinsic signals will not be discussed further; they are the subject of other reviews (e.g. Grinvald et al. 1988).

\section{TWO-PHOTON SCANNING MICROSCOPY IMPROVES SPATIAL RESOLUTION}

Using one-photon, wide-field microscopy, brain images are blurred by light scattering and out-offocus fluorescence. Figure $5 a$ shows an example of such an image of a mouse olfactory bulb. In this preparation, the incoming olfactory receptor nerve terminals have been stained with a calcium dye, Calcium Green-1 dextran. In this image, individual glomeruli can just be made out as the spatial resolution is approximately $50 \mu \mathrm{m}$. It would be impossible to discern the images of individual juxtaglomerular neurons that surround each of the glomeruli. In striking contrast, figure $5 b$ illustrates a two-photon measurement showing a portion of an individual glomerulus surrounded by the easily identified cell bodies of juxtaglomerular neurons. Clearly, two-photon microscopy has provided a large improvement in spatial resolution. The two-photon spatial resolution is also superior to (a) Voltage vs calcium

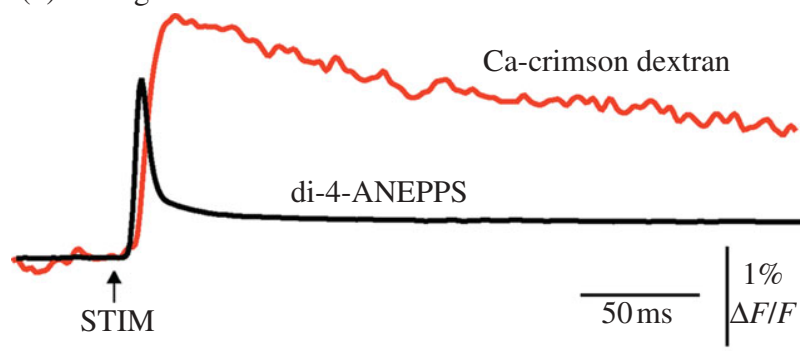

(b) Calcium vs intrinsic signals

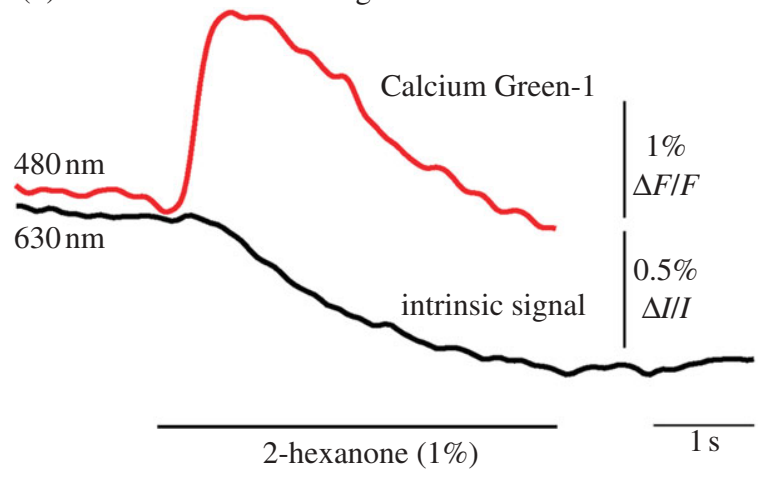

Figure 4. Comparisons of voltage, calcium, and intrinsic imaging signals. (a) A comparison of the time course of a voltage and a calcium dye signal from the same location near the merger of the olfactory nerve and the olfactory bulb in an in vitro turtle preparation. The voltage signal is faster. (M. Wachowiak \& L. B. Cohen, unpublished.) (b) A comparison of the time course of the calcium signal and the intrinsic imaging signal in a mouse olfactory bulb glomerulus in response to the odorant, 2-hexanone. The intrinsic imaging signal is substantially slower than the calcium signal. (Modified from Wachowiak \& Cohen 2003.)

confocal microscopy where the effect of light scattering and out-of-focus light are reduced by measuring only the emitted photons that pass back through the illumination pinhole. Using confocal microscopy, it is difficult to obtain cellular resolution at depths greater than $50 \mu \mathrm{m}$ although, for cells or processes close to the surface, confocal microscopy is successful. Using a scanned-line confocal microscope from Prairie 


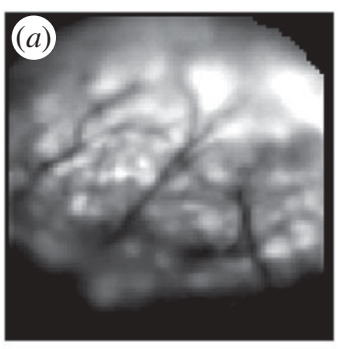

100 frames averaged

$$
\text { 2-photon } \quad 20 \bar{\mu} \mathrm{m}
$$
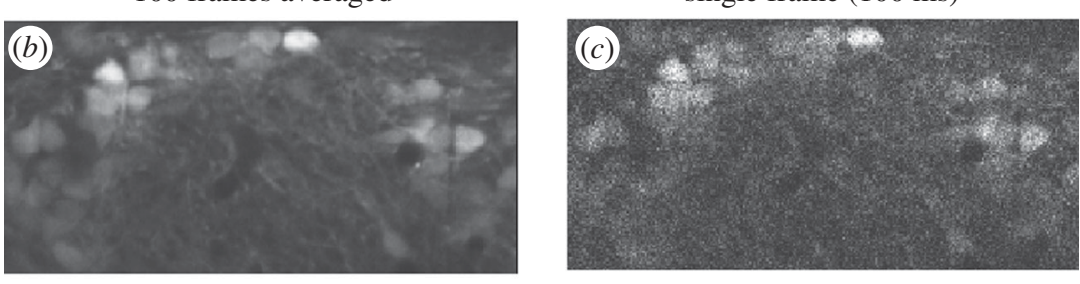

$100 \mu \mathrm{m}$

Figure 5. Comparison of the spatial resolution of wide-field and two-photon imaging from the mouse olfactory bulb. (a) Onephoton wide-field image of the bulb. Individual glomeruli can be recognized but they are substantially blurred as a result of light scattering. $(b, c)$ Two-photon: bolus loading with Oregon Green 488 BAPTA 1 AM. (b) A two-photon image from a $10 \mathrm{~s}$ recording. A part of a glomerulus is surrounded by easily recognized juxtaglomerular neurons. The spatial resolution is dramatically better than the one-photon wide-field recording (100 frames averaged; scale bar, $100 \mu \mathrm{m}$ ). (c) The same as (b) but the recording time is reduced to $0.1 \mathrm{~s}$ (single frame; $100 \mathrm{~ms}$ ). Because the number of measured photons has been reduced by a factor of 100 the relative shot noise is increased by a factor of 10 and the image is blurred.

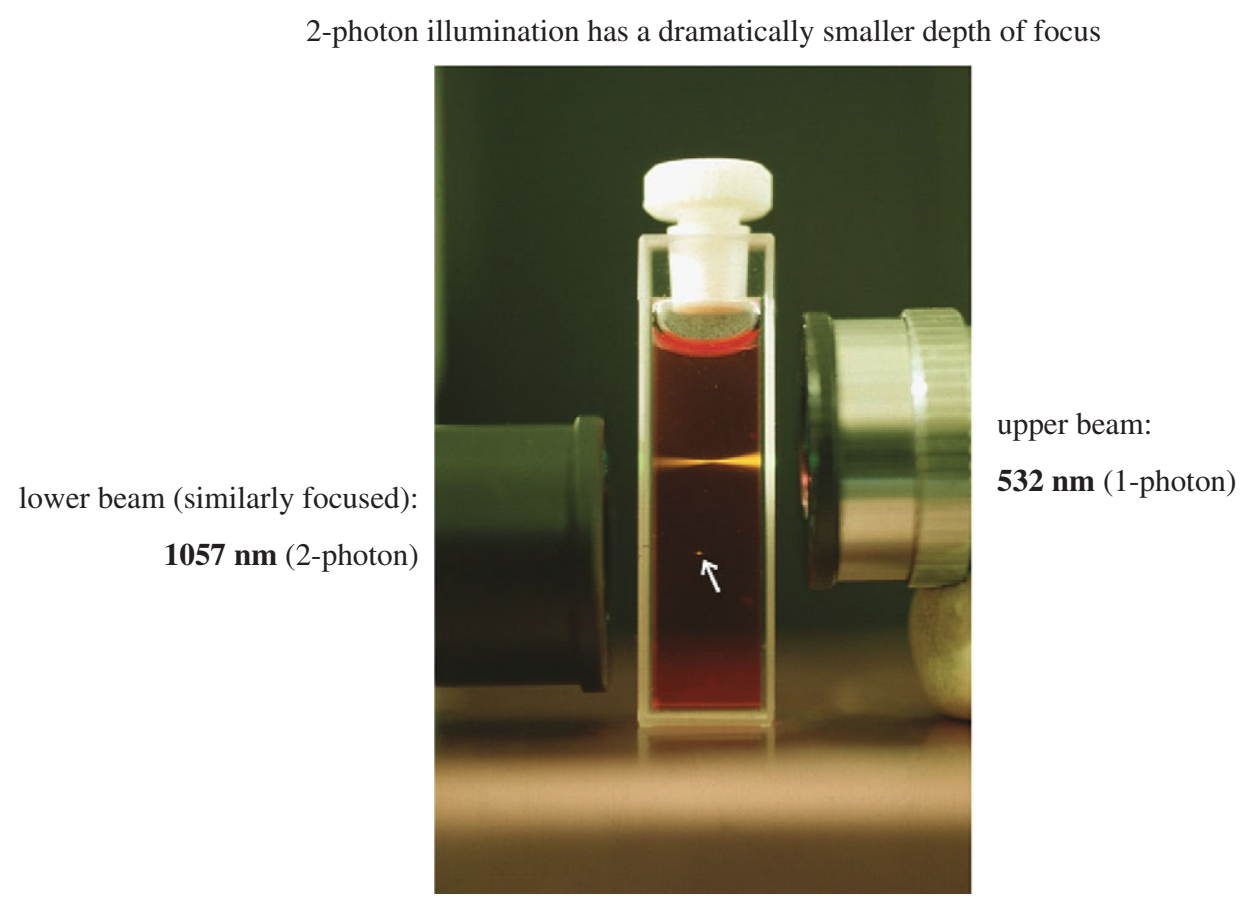

Figure 6. Illumination with one-photon and two-photon wavelengths. With the one-photon beam (532 nm) coming from the right there is fluorescence along the entire light path. With the two-photon wavelength $(1057 \mathrm{~nm})$ coming from the left, fluorescence is excited only at the small focal spot indicated by the arrow. This spot can be raster scanned over the object and the fluorescence from each location measured; knowing the location of the spot and the intensity allows an image to be reconstructed. (Brad Amos, Science Photo Library, London).

Technologies (Middletown, WI, USA), Jeff Magee (personal communication) were able to measure voltage-sensitive dye signals from hippocampal cell dendrites at a frame rate of $3 \mathrm{kfps}$. Slower voltagesensitive dye signals were measured confocally much earlier (Loew 1993).
The demonstration illustrated in figure 6 can be used to understand the improved spatial resolution in twophoton microscopy. There are two incident light beams directed at the cuvette filled with a fluorescent dye. The beam coming from the right is at $532 \mathrm{~nm}$, a wavelength at which each photon can excite the dye. 

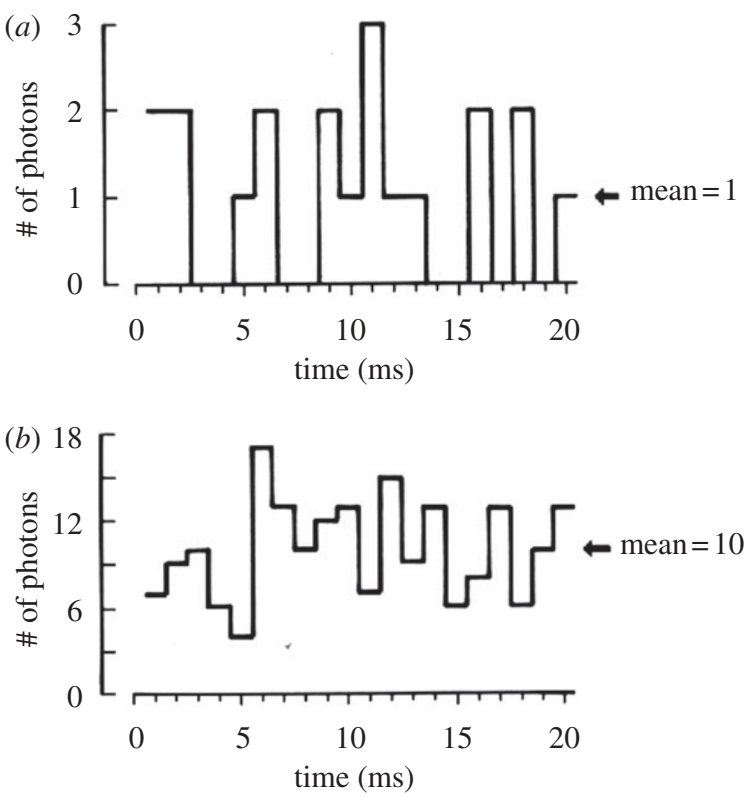

Figure 7. Signal-to-noise ratio: the relative noise decreases (the signal-to-noise ratio increases) when more photons are measured. Plots of the results of using a table of random numbers to distribute 20 photons (a) or 200 photons (b) into 20 time bins. When more photons are measured the signal-to-noise ratio is improved.

This beam excites fluorescence all along its light path. The incident light beam coming from the left is at twice the wavelength, and two of these photons have to arrive simultaneously in order to excite the dye. In this case, fluorescence is seen only in a tiny spot in the middle of the cuvette (indicated by the arrow). This shallow depth of illumination is the expected result for the case where fluorescence excitation is proportional to the square of light intensity. In out-of-focus regions, the intensity will be lower, but the square of intensity much lower, than at the focal point. With two-photon illumination, fluorescent photons are only generated at the focal point; therefore their source is known. Even though most of the emitted photons will be scattered by the brain, because their source is known, two-photon microscopes attempt to maximize the collection of the emitted photons.

In spite of efforts that are made to collect and measure as many emitted photons as possible, the number of measured photons remains small. Perhaps a better name for this technique would be 'few photon microscopy'. The fact that only a few photons are measured means that the relative shot noise will be large and the signal-to-noise ratio will be low (see the following).

\section{NOISE IN OPTICAL MEASUREMENTS}

A noiseless optical measurement is rarely encountered (however, see figure $8 b$ ). In the three examples given in the following, the fractional intensity changes can be quite small; they range from $2 \times 10^{-4}$ to $5 \times 10^{-1}$. To measure these signals, the noise in the measurements had to be even smaller. In the sections that follow, some of the considerations necessary to achieve such a low noise are outlined.
Table 1. Photons $\mathrm{ms}^{-1}$ pixel $^{-1}$

\begin{tabular}{llc}
\hline & imaging method & $\begin{array}{l}\text { photons ms }^{-1} \\
\text { pixel }^{-1}\end{array}$ \\
\hline cell body & $\begin{array}{l}\text { two-photon } \\
\text { fluorescence } \\
\text { distal dendrite } \\
\text { wide-field } \\
\text { fluorescence, } \\
\text { intracellular dye, arc } \\
\text { lamp }\end{array}$ & 1 \\
brain slice & \begin{tabular}{l} 
wide-field absorption \\
\hline
\end{tabular} & 1000 \\
\hline
\end{tabular}

There are many types of extraneous (technical) noise, including noise from floor vibrations, room air currents, unstable light sources, detector read noise, detector dark noise and, in in vivo preparations, heart-beat or respiration movements. Reducing the contribution of these sources of noise requires careful attention to the details of the apparatus (Grinvald et al. 1988; Zochowski et al. 2000; Homma et al. 2009; www.redshirtimaging.com/redshirt_neuro/neuro_lib_ 4.htm) but with care, extraneous noise can often be reduced to a level smaller than that of the shot noise.

\section{(a) Shot noise}

The limit of accuracy with which light can be measured is set by the shot noise arising from the statistical nature of photon emission and detection. With currently available light sources, there are random fluctuations in the number of photons emitted (and measured) per unit time; the root-mean-square (r.m.s.) deviation in the number measured is the square root of the average number (Braddick 1960; Malmstadt et al. 1974). Thus, in a shot-noise-limited measurement, the signal-to-noise ratio is directly proportional to the square root of the number of measured photons and inversely proportional to the square root of the bandwidth of the photodetection system. As a result, attempts to increase the number of measured photons exert a powerful driving force on the design of all parts of an optical recording system.

The basis for the square root dependence on intensity is illustrated in figure 7 . In figure $7 a$ the result of using a random number table to distribute 20 photons into 20 time windows is shown. In figure $7 b$ the same procedure was used to distribute 200 photons into the same 20 bins. Relative to the average light level, 1 in figure $7 a$ and 10 in figure $7 b$, there is more noise in the top trace than in the bottom trace. The signal to r.m.s. noise level is 1 in figure $7 a$ and 3 in figure $7 b$, close to the expected square root dependence on light intensity.

A very large range of measured intensities is obtained in full frame (10 000-100 000 pixels) optical recordings from the nervous system (table 1). This range is affected both by the preparation and by the imaging method.

Needless to say, the very small number of photons per millisecond in a two-photon measurement has powerful consequences. First, one never sees fullframe two-photon measurements with a $1 \mathrm{kHz}$ frame 
(a) 2-photon microscope

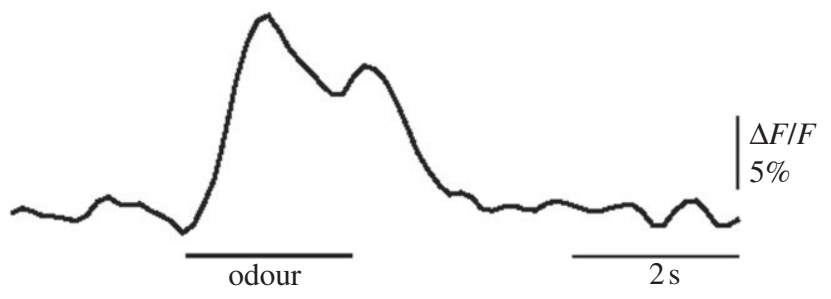

(b) ordinary microscope

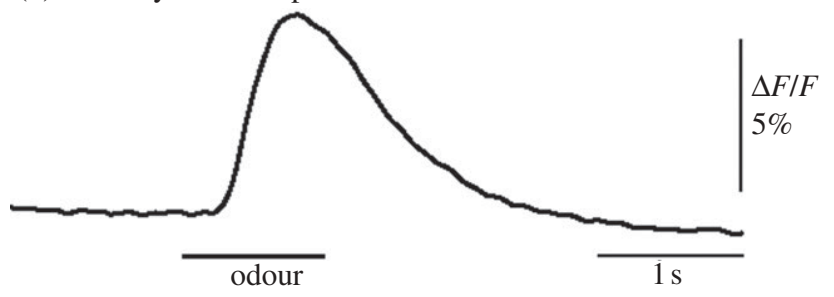

(c) 2-photon microscope

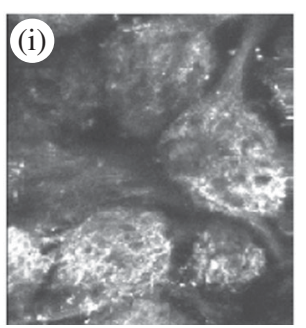

ordinary microscope

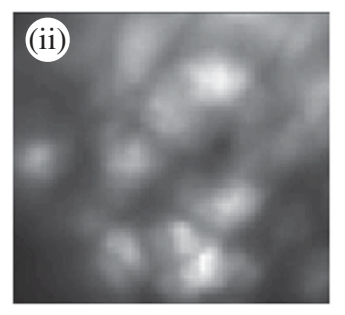

Figure 8. Calcium Green-I signals: comparison of a twophoton and a one-photon measurement from the same glomerulus in an in vivo mouse preparation responding to an odorant. (a) The two-photon measurement is noisy (two-photon microscope; 4 trials). (b) The one-photon wide-field measurement has a much larger signal-to-noise ratio (ordinary microscope with a NeuroCCD-SM camera; 1 trial). In both measurements the light from all of a glomerulus was spatially averaged. (c) In contrast, in images the spatial resolution of the two-photon measurement (i) is far superior to the one-photon measurement (ii).

rate. The result in figure $5 b$ was the average of 100 frames; a total of $10 \mathrm{~s}$ of recording. Figure $5 c$ shows one of the individual frames from a $100 \mathrm{~ms}$ recording period. The individual $100 \mathrm{~ms}$ recording is substantially blurred compared with the $10 \mathrm{~s}$ recording. Clearly, a $1 \mathrm{~ms}$ recording would be dominated by the blur of shot noise. Thus, more commonly used frame rates for activity measurements are $5-10 \mathrm{~Hz}$ and at that frame rate the r.m.s. noise is still 10 per cent of the signal. A further result of the small number of photons is that much attention has to be devoted to optimizing the incident light intensity, the collection of emitted photons and the quantum efficiency of the photodetector in two-photon measurements.

The modest number of photons in wide-field measurements from a dendrite means that cameras with low read and dark noise are needed to avoid their contribution to the total noise. For that reason, CCD cameras with their relatively low read noise are preferred over complimentary metal-oxide semiconductor (CMOS) cameras. On the other hand, for wide-field brain slice experiments with a large number of photons a CMOS camera with very deep wells is required to avoid the saturation that would occur with a CCD camera.

\section{(i) The optimum signal-to-noise ratio in two-photon microscopy}

Because two-photon excitation will only occur with the nearly synchronous arrival of two low-energy photons, excitation is proportional to the square of light intensity and only very high intensity sources achieve significant excitation. In practice, this restricts the light source to pulsed lasers with very brief (approx. $100 \mathrm{ps}$ ), but very high intensity pulses. In the experiments illustrated in the following, the intensity available from commercial lasers was not limiting because the targeted cells were only some $20-200 \mu \mathrm{m}$ below the surface of the preparation and the losses in focal plane intensity from light scattering were not large. However, the incident intensity could not be increased further to increase the number of measured photons and improve the signal-to-noise ratio because higher intensities would cause tissue heating resulting from the non-zero absorption of infrared photons. For cells as deep as rodent olfactory bulb mitral cells (approx. $400 \mu \mathrm{m}$ below the surface), the loss of excitation light because of photon dispersion within the tissue becomes large, and the spatial resolution starts to be limited by out-of-focus fluorescence that is generated as a result of increasing the laser power (Helmchen \& Denk 2005). In the brainstem one might expect to be able to record the activity of neurons up to $300 \mu \mathrm{m}$ below the surface using currently available dyes and apparatus.

\section{(ii) The optimum signal-to-noise ratio in a wide-field measurement}

A tungsten filament lamp emits an average of $10^{16}$ photons $\mathrm{ms}^{-1}$ and the r.m.s. deviation in the light intensity is the square root of this number or $10^{8}$ photons $\mathrm{ms}^{-1}$. However, because only a small fraction of the photons will be measured, a signal-to-noise ratio of $10^{8}$ (see above) cannot be achieved. A partial listing of the light losses follows. A 0.9-NA lamp collector lens would collect 0.1 of the light emitted by the source. Only 0.2 of that light is in the visible wavelength range; the remainder is infrared (heat). Limiting the incident wavelengths to those that excite the fluorescence means that only 0.1 of the visible light is used. Thus, the light reaching the preparation might typically be reduced to $10^{13}$ photons $\mathrm{ms}^{-1}$. If the light-collecting system that forms the image has high efficiency, e.g. in an absorption measurement with matched condenser and objective numerical apertures, approximately $10^{13}$ photons $\mathrm{ms}^{-1}$ will reach the image plane. (In a fluorescence measurement there will be much less light measured because (i) in an individual cell less than 10 per cent of the incident photons are absorbed by the fluorophores, (ii) only a fraction of the absorbed photons will result in emitted photons, and (iii) only a fraction of the emitted photons are collected by the objective.) If the camera has a quantum efficiency of 1.0, then, in absorption, a total of $10^{13}$ photoelectrons $\mathrm{ms}^{-1}$ will be measured. With a camera of 10000 pixels, there will be $10^{9}$ photoelectrons $\mathrm{ms}^{-1}$ pixel $^{-1}$. The shot noise will be 
Voltage imaging of activity in the dendrites of a single mitral cell

(a)

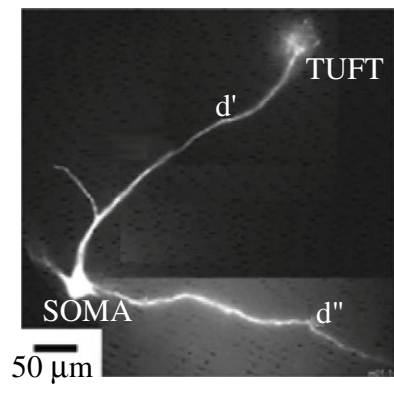

(b)

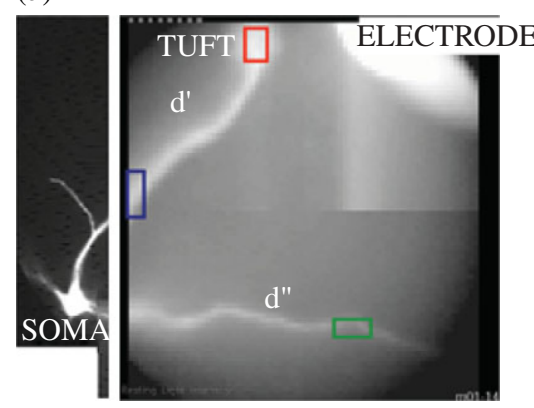

(c)

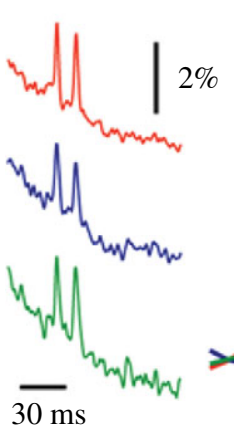

(d)

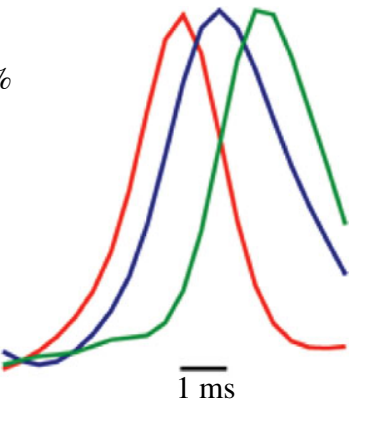

(e)

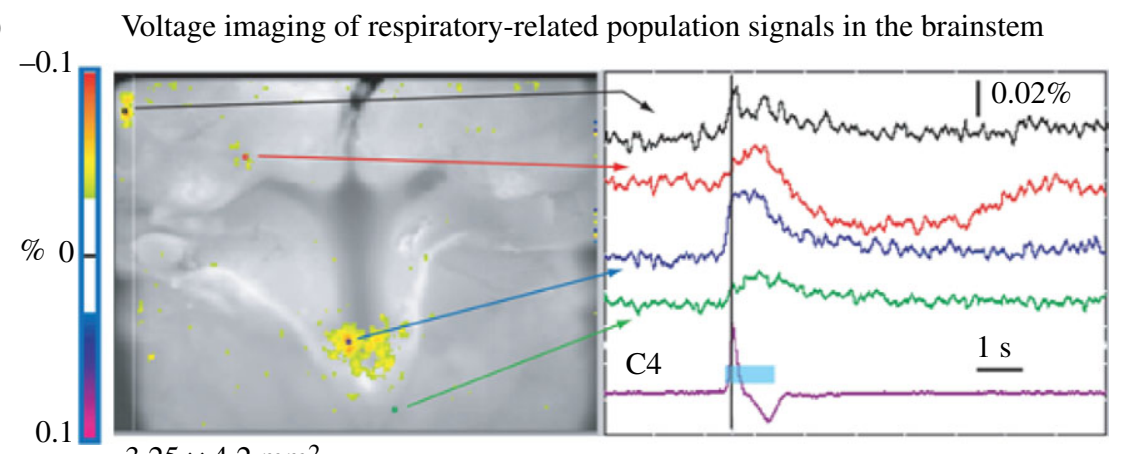

$3.25 \times 4.2 \mathrm{~mm}^{2}$

Figure 9. Caption opposite.

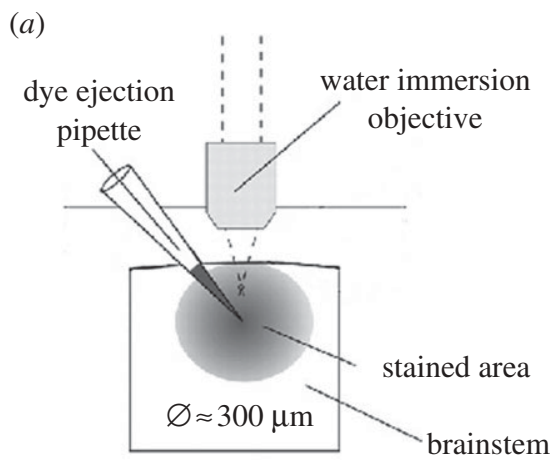

(b) Resting light intensity (grey scale)

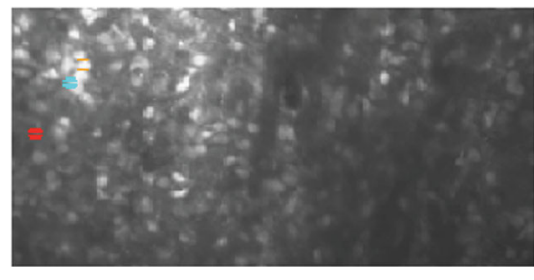

(d) Frame subtraction (during-before; pseudo-colour)

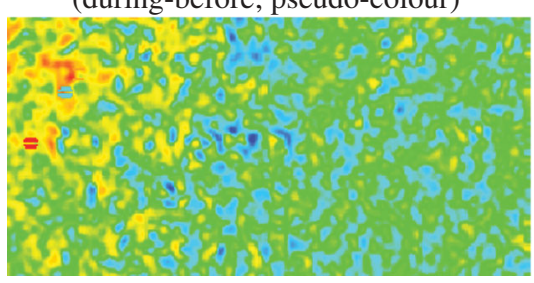

(c)

Oregon Green 2-photon calcium signals from three cells
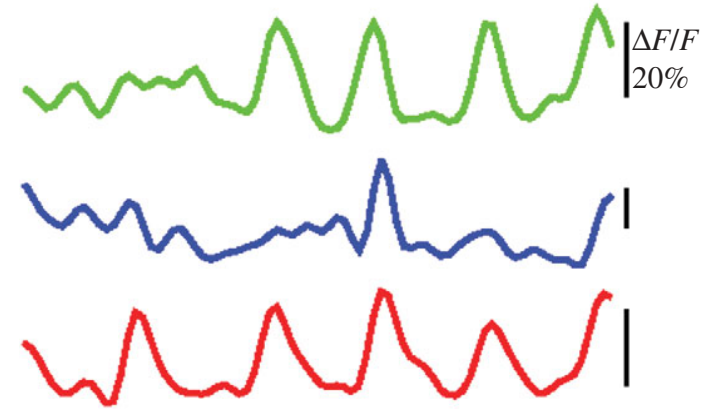

C5 electrode recording

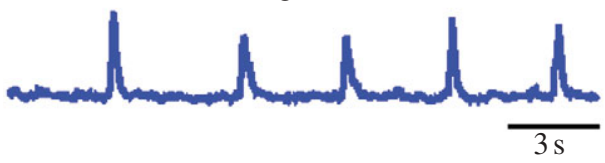

Figure 10. Caption opposite. 
Figure 9. (Opposite.) Voltage-sensitive dye measurements made with wide-field illumination. ( $a-d$ ) Measurements from the dendritic processes of a single mitral cell in an olfactory bulb slice preparation. The dye was intracellulary injected using a patch pipette. Two action potentials were elicited by extracellular stimulation. The first action potential originated in the tuft in the glomerulus and propagated throughout the cell. (Modified from Djurisic et al. 2004). (e) Population signals from a rat neonatal brainstem preparation during respiratory activity. Disparate regions have activity linked to inspiration; the time course of the activity differs in the different regions. (Modified from Onimaru \& Homma 2005.)

$10^{4.5}$ photoelectrons $\mathrm{ms}^{-1}$ pixel $^{-1}$; thus the very best that can be expected is a noise that is $10^{-4.5}$ of the resting light (a signal-to-noise ratio of approx. $90 \mathrm{db}$ ). The extra light losses in a fluorescence measurement would further reduce the maximum obtainable signal-tonoise ratio.

\section{A DIRECT COMPARISON OF ONE- AND TWO- PHOTON IMAGING OF GLOMERULAR ACTIVITY}

From the preceding discussion of the sources of noise in an activity measurement one would predict that the signal-to-noise ratio would be larger in a one-photon wide-field measurement than in a two-photon measurement. The results illustrated in figure 8 show that this is the case. In figure $8 a$ a two-photon recording of activity from a glomerulus in a mouse olfactory bulb is shown. Figure $8 b$ shows the one-photon recording from the same glomerulus. The one-photon measurement has a much larger signal-to-noise ratio.

Moreover, four trials were averaged in the two-photon measurement while the result from the one-photon measurement was from a single trial. In addition, the numerical aperture of the lens used for the two-photon measurement was 0.8 while that used in the ordinary microscope was only 0.5 . If a correction for these two factors is applied, the two-photon measurement would have a signal-to-noise ratio five times smaller than that shown. Thus, the signal-tonoise ratio for the wide-field measurement is orders of magnitude better.

In contrast to the advantage in signal-to-noise ratio of the one-photon measurement is the dramatically superior spatial resolution of the two-photon measurement. This is illustrated in figure $8 c, d$. The glomeruli are a blur in the one-photon measurement, while considerable internal detail is seen in the two photon measurement.

\section{THREE EXAMPLES}

(a) One-photon voltage dye imaging of activity in dendrites of an individual neuron

Understanding the biophysical properties of single neurons and how they process information is fundamental to understanding how the brain works. At present, however, the detailed functional structure of nerve cells is not fully understood. Part of the explanation for this incomplete understanding is the complexity of the neuronal structure and membrane properties (figure $1 a$ ). It is widely recognized that dendritic membranes of vertebrate CNS neurons contain many active conductances such as voltage-activated $\mathrm{Na}^{+}, \mathrm{Ca}^{2+}$ and $\mathrm{K}^{+}$channels (e.g. Stuart \& Sakmann 1994; Sjostrom et al. 2008). As a result, the regional electrical properties of branching neuronal processes will be extraordinarily complex, dynamic and, in the general case, impossible to predict in the absence of detailed measurements. To obtain such a measurement, the sensitivity of the intracellular voltage-sensitive dye technique has been dramatically improved, allowing direct recording of subthreshold and action potential signals from the neurites of individual neurons (Zecevic 1996; Antic 2003; Canepari et al. 2007). The improved sensitivity depends on using fluorescence measurements and selective staining of only one neuron within a preparation (Grinvald et al. 1988). Another important advance was finding a dye that gives relatively large signals when applied internally. The fluorescent voltage-sensitive styryl dye JPW 1114 (also called di-2ANEPEQ; Invitrogen-Molecular Probes Inc., D6923) is currently an optimal voltage indicator for intracellular application aimed at recording from neuronal processes of individual neurons in brain slices (figure 9; Antic 2003; Canepari et al. 2007).

This dye had little or no pharmacological effect when applied at functional concentrations to both invertebrate (Antic et al. 2000) and vertebrate neurons (Canepari et al. 2007). Similarly, photodynamic damage during optical recording was not present if the exposures of the dendritic arbour to excitation light were kept relatively short $(100 \mathrm{~ms}$; Djurisic et al. 2004, 2008; Canepari et al. 2007).

In the example shown in figure $9 a-d$, a stationarystage upright microscope with a $250 \mathrm{~W}$ xenon, short-gap arc lamp (Osram, XBO $250 \mathrm{~W} / \mathrm{CR}$ ORF) powered by a low ripple power supply was used. The fluorescence signals were recorded using a fast camera with relatively low spatial resolution $(80 \times 80$ pixels) but outstanding dynamic range and low read noise (NeuroCCD-SM; RedShirtImaging LLC, Decatur, GA, USA).

Figure $9 a-d$ illustrates the recording of action potentials from the dendrites of a mitral cell in an

Figure 10. (Opposite.) Calcium signals from individual neurons correlated with inspiration in an embryonic mouse brainstemspinal chord preparation. (a) A schematic illustrating multi-cell bolus loading of neurons with a calcium-indicator dye. The membrane-permeant dye is pressure-injected from the patch pipette into the extracellular space. Subsequently it diffuses into the cells where it is de-esterified by intracellular esterases. (b) Resting light intensity; grey scale. The image of calcium fluorescence from stained neurons. (c) Calcium signals from three neurons (top three traces) indicated by the small coloured circles in $(b)$. The bottom trace in $(c)$ is the integrated electrode recording from spinal root C5. (d) A colour-coded subtraction image of three frames recorded before a response from three frames recorded during a response. This subtraction image shows the locations of calcium increases (indicated in red). The activity of many $(>10)$ cells was recorded from this plane. (J. Eugenin, J. G. Nicholls, L. B. Cohen, B. Lindsey, R. Homma \& K. Muller, unpublished observations.) 
olfactory bulb slice. The mitral cell was stimulated by shocking the slice with an extracellular electrode in the olfactory nerve layer (figure $9 b$ ). This stimulus elicited two spikes (figure $9 c$ ) that were recorded optically from the dendritic structure projected onto the CCD. The spatial average of the action potential signals from three colour-coded regions are shown in figure $9 b, c$. When the recording of the first action potential is displayed on an expanded time scale (figure $9 d$ ), it is clear that the action potential originates in the dendritic tuft in the glomerulus and then propagates to the soma and out the secondary dendrite. The action potential actively invades all of the dendrites (Djurisic et al. 2004).

\section{(i) Attractive features of this kind of measurement}

The ability to follow changes in membrane potential throughout the dendritic and axonal arbour of a neuron can provide critical information about neuron function. Recent improvements in the technique have allowed recordings of action potentials from individual dendritic spines (D. Zecevic 2008, unpublished data).

\section{(ii) Unattractive features}

One difficulty is that this kind of recording is thus far limited to in vitro brain slice preparations. The results might be qualitatively different in the presence of an in vivo milieu containing additional transmitters and modulators.

\section{(b) One-photon voltage imaging of population signals from the brainstem during respiratory activity}

In a number of situations (figure $1 c$ ), signals recorded from populations of neurons can be informative even though these signals do not represent the activity of individual cells but the average of the activity of many cells and processes whose light reaches the individual pixels in the recording camera. Examples of informative measurements are the calcium signals from olfactory receptor nerve terminals in the olfactory bulb (Wachowiak \& Cohen 2001) and orientation-sensitive intrinsic signals from visual cortex (Bonhoeffer \& Grinvald 1993). Similarly, respiratory activity in the brainstem occurs in many distinct regions over a large area. Onimaru \& Homma (2005) isolated a brainstem-spinal chord preparation from neonatal rats and superfused the preparation with the styryl voltage-sensitive dye, di-2-ANEPEQ. Using a MiCAM01 CCD camera (BrainVision, Tokyo, Japan), they recorded the fluorescence from the dorsal surface during 20 respiratory cycles and then aligned the 20 recordings using the inspiratory activity recorded electrically from the spinal root C4. Figure $9 e$ shows the results of such a recording. The left panel is an image of the preparation on which is superimposed a thresholded pseudo-colour map of the location of the signals recorded at the time of the vertical line in the right panel. The pseudo-colour indicates obvious signals in two locations (the black and blue traces on the right panel). Slower signals were recorded at two other locations, red and green on the right panel. In addition, one location, red, had a large late hyperpolarizing signal. Thus, the voltage imaging enabled a quick discovery and localization of regions in the dorsal brainstem with activity related to respiration.

\section{(i) Attractive features of this kind of measurement}

The method offers considerable power for localizing the activity related to respiratory behaviour. Because a voltage-sensitive dye was used, the signal is known to represent changes in membrane potential.

\section{(ii) Unattractive features}

The technique is restricted to the upper layers of the preparation because the incident light does not reach deeper layers and fluorescence photons from deeper layers do not escape from the tissue. Light scattering and out-of-focus light limit the spatial resolution. Averaging of many trials was required to improve the signal-to-noise ratio obscuring variations in response from cycle to cycle. The organic voltage-sensitive dye that was used stains all membranes, so the neuronal or glial cell types responsible for the signals are not known. Thresholding was used in the left panel of figure $9 e$; if a different threshold was chosen or more spatial or temporal filtering was used, the results would be qualitatively different.

\section{(c) Two-photon measurement of activity of many individual neurons in the brainstem during respiratory cycling}

Nervous systems are made up of large numbers of neurons, and many of these are active during the generation of behaviours (figure $1 b$ ). The original motivation for developing optical methods for monitoring activity was the hope that they could be used to record the activities of many neurons simultaneously during behaviours (Davila et al. 1973). One simple and widely used method for monitoring neuronal activity relies on imaging the neuron's intracellular $\mathrm{Ca}^{2+}$ concentration. Indeed, in living cells, most depolarizing electrical signals are associated with a $\mathrm{Ca}^{2+}$ influx caused by the activation of different types of voltage-gated $\mathrm{Ca}^{2+}$ channels (Tsien \& Tsien 1990; Berridge et al. 2000). Such signals can be further amplified by $\mathrm{Ca}^{2+}$ release from intracellular $\mathrm{Ca}^{2+}$ stores (Verkhratsky 2005). The easiest technique to monitor the activities of many individual neurons by imaging their intracellular $\mathrm{Ca}^{2+}$ concentration uses a membrane-permeant acetoxymethyl (AM) ester form of a $\mathrm{Ca}^{2+}$ indicator dye (Tsien 1981). For the vertebrate brain, the method allowing imaging of neural ensembles in with single cell resolution was introduced by Stosiek et al. (2003; for experimental details see Garaschuk et al. 2006). The technique (figure 10a) has been successfully applied to different neuronal tissues in a variety of species from lower vertebrates to mammals (Brustein et al. 2003; Nimmerjahn et al. 2004; Kerr et al. 2005; Li et al. 2005; Niell \& Smith 2005; Ohki et al. 2005; Sullivan et al. 2005). This technique uses a 'bolus' injection of a membrane-permeant $\mathrm{Ca}^{2+}$ indicator dye into the extracellular space. A pipette containing the AM form of an indicator dye is inserted into the tissue of interest and approximately $400 \mathrm{fl}$ of the 
dye-containing solution is pressure ejected. The dye diffuses into the cells of interest where it is hydrolyzed by intracellular esterases and thereby trapped internally. The remaining AM dye is rapidly removed from the extracellular space by the microcirculation.

Figure $10 b$ illustrates the resting fluorescence from the calcium dye that has filled many cells in the embryonic brainstem. Some cells are brighter and some neighbours are less bright. We do not know whether this reflects differences in dye concentration or differences in resting free calcium concentration, or both. There are three coloured spots superimposed on this image. The traces of light intensity versus time from these three cells are shown as the top three traces in figure $10 c$. The bottom trace in figure $10 c$ is the integrated suction electrode recording from a C5 spinal root. One of the cells (red) is active during each of the five respiratory cycles; one (green) is active in four of the five cycles; and one (blue) seems to be active in only two of the cycles (J. Eugenin et al. 2008, unpublished data.).

Figure $10 d$ shows a subtraction of frames recorded prior to a response from frames recorded during a response where red colour indicates the location of a calcium increase during the response. This framesubtraction image shows the location of many calcium signals. Clearly, a large number of cells were active during these respiratory cycles.

(i) Attractive features of this kind of measurement

The method allows one to measure the activity of many cells simultaneously. Averaging of trials was not required, and thus breath-to-breath response changes could be detected.

\section{(ii) Unattractive features}

The technique is restricted to the upper layers of the preparation because the incident light does not reach deeper layers. The organic calcium-sensitive dye that was used stains all cells, so the cellular origins of the signals are not known. Because a calcium-sensitive dye was used, the source of the signal is not certain; it might arise from calcium influx through voltage-sensitive calcium channels or from release from internal stores. The signal-to-noise ratio is not large; signals smaller than $5-10 \%$ will be missed, and thus the recordings are likely to be incomplete.

\section{THE AMPLITUDE OF THE VOLTAGE OR CALCIUM CHANGE IS DIFFICULT TO DETERMINE}

Both voltage and calcium signals are often presented as a fractional intensity change $(\Delta I / I)$. These signals give information about the time course of the potential or calcium-concentration change but no direct information about the absolute magnitude. However, in some instances, approximate estimations can be obtained. For example, the size of the optical signal in response to a sensory stimulus can be compared with the size of the signal in response to an epileptic event (Orbach et al. 1985). Another approach is the use of ratiometric measurements at two independent wavelengths (Grynkiewicz et al. 1985; Gross et al. 1994). However, to determine the amplitude of the voltage or calcium change from a ratio measurement, one must know the fraction of the fluorescence that results from dye in the expected location, i.e. bound to active versus inactive membranes for voltagesensitive dyes, or dye free in the axoplasm versus dye bound to protein or in intracellular compartments for calcium dyes. These requirements are only approximately met in special circumstances.

\section{METHODOLOGICAL CONSIDERATIONS REGARDING APPARATUS}

\section{(a) Numerical aperture}

The need to maximize the number of measured photons has been a dominant factor in the choice of optical components. In wide-field epifluorescence, both the excitation light and the emitted light pass through the objective, and the intensity reaching the photodetector is proportional to the fourth power of the numerical aperture (NA; Inoue 1986). In twophoton imaging, only the collection of emitted light is affected by numerical aperture, and thus the intensity reaching the photodetector is proportional to the square of the numerical aperture.

\section{(b) Back aperture of the objective}

Because of the strong light scattering within living tissue, in vivo two-photon imaging critically depends on detection of scattered photons. Under this condition, the size of the back aperture of the objective (but also the size of all other apertures within the detection pathway) becomes important. Ideally, one would like to employ an objective with high NA, large field of view (low magnification) and large back aperture. More optimized objectives recently became available.

\section{(c) Depth of focus in wide-field imaging}

Salzberg et al. (1977) determined the effective depth of focus for a $0.4 \mathrm{NA}$ objective lens by recording an optical signal from a neuron when it was in focus and then moving the neuron out of focus by various distances. They found that the neuron had to be moved $300 \mu \mathrm{m}$ out of focus to cause a 50 per cent reduction in signal size. Using 0.5 NA optics, Kleinfeld \& Delaney (1996) found that $100 \mu \mathrm{m}$ out of focus led to a reduction in signal size of 50 per cent.

\section{(d) Light scattering and out-of-focus light in wide-field imaging}

Light scattering and out-of-focus light limit the spatial resolution of a wide-field optical measurement from a thick scattering tissue like the brain. With a $0.4 \mathrm{NA}$ objective, moving a small spot of light out of focus will increase its diameter to approximately $200 \mu \mathrm{m}$. Similarly, a small spot of light will be blurred to a diameter of approximately $200 \mu \mathrm{m}$ after it passes through $500 \mu \mathrm{m}$ of salamander olfactory bulb. (Mammalian cortex appears to scatter more than the salamander olfactory bulb.) Presumably this effect will be more severe at lower wavelengths because scattering by nervous tissue is inversely related to wavelength squared (Cohen \& Keynes 1971). 


\section{(e) Electron-multiplying charge-coupled device cameras}

These cameras have on-chip multiplication and should lead to better signal-to-noise performance at very low light levels. However, the multiplication process adds noise (a factor of 1.4) and some existing chips are even noisier than expected from the factor of 1.4. If an ordinary CCD has an r.m.s. read noise of 10e-, then an ideal EM-CCD camera will have a better signal-to-noise ratio only at light levels less than 100 photons pixel $^{-1}$ frame $^{-1}$. The light level achieved in the experiment on the dendrites of a single neuron (figure $9 a-d$ ), where there is relatively little dye in small distal processes, is approximately 1000 photons $/$ pixel $/ \mathrm{ms}$. Thus in this measurement, and all measurements with even higher light intensity, a CCD camera with a read noise of $10 \mathrm{e}-$ will have a better signal-to-noise ratio than an EM-CCD camera.

\section{FUTURE DIRECTIONS}

\section{(a) Organic voltage-sensitive dyes}

The voltage-sensitive dyes in figure 2 and the vast majority of those synthesized are of the general class named polyenes (Hamer 1964), a chemical group that was first used to extend the wavelength response of photographic film. It is possible that improvements in signal size can be obtained with new polyene dyes (see Waggoner \& Grinvald 1977; Fromherz et al. 1991 for a discussion of maximum possible fractional changes in absorption and fluorescence). On the other hand, the maximum fractional change has not increased in recent years, and most improvements (e.g. Grinvald et al. 1982; Antic \& Zecevic 1995; Momose-Sato et al. 1995; Tsau et al. 1996) have involved synthesizing analogues that work well on new preparations.

The best of the organic dyes have fluorescence changes of $10-20 \%$ per $100 \mathrm{mV}$ in situations where the staining is specific to the membrane whose potential is changing (Grinvald et al. 1982; Loew et al. 1992; Rohr \& Salzberg 1994). Gonzalez \& Tsien (1995) introduced a new scheme for generating voltagesensitive signals using two chromophores and fluorescence resonance energy transfer (FRET). While these fractional changes were also in the range of 10 per cent per $100 \mathrm{mV}$, more recent results are approximately 30 per cent (J. Gonzalez and R. Tsien 1997, personal communication). However, in order to achieve fast response times $(>100 \mathrm{~Hz})$, one of the chromophores must be very hydrophobic and as a result does not penetrate into brain tissue. Thus far it has not been possible to measure signals with a fast pair of dyes in intact tissues ( $\mathrm{T}$. Gonzalez, R. Tsien, A. Obaid \& B. M. Salzberg 2000, personal communication), although impressive results have been obtained where speed was not critical (Cacciatore et al. 1999; Briggman \& Kristan 2006).

Membrane potential changes the nonlinear second harmonic generation from styryl dyes (Bouevitch et al. 1993; Dombeck et al. 2005; Millard et al. 2005). Large (50\%) fractional changes were measured. But, because the number of detected photons is small even compared with two-photon microscopy, the signal-to-noise ratios are very small.

\section{(b) Neuron-type-specific staining}

An important new direction is the development of methods for neuron-type-specific staining, which would make it possible to determine the role of specific neuron types in generating the input-output function of a brain region. Two quite different approaches have been tried.

\section{(i) Directed transport}

The use of anterograde and retrograde dye transport has resulted in specific staining of olfactory receptor neuron terminals (Friedrich \& Korsching 1997; Wachowiak \& Cohen 2001) and motor neurons in embryonic chicks and in lamprey spinal cords (Tsau et al. 1996). For the anterograde direction, this method depends on finding a location where only one cell type is present that has a process leading to the brain area of interest. For the retrograde direction, this method depends on finding a fibre tract that comes from a single neuron type.

\section{(ii) Genetically encoded activity sensors \\ Voltage sensors}

Siegel \& Isacoff (1997) constructed a genetically encoded combination of a potassium channel and green fluorescent protein. When expressed in a frog oocyte, this molecule, FlaSh, had a (relatively slow; approx. $100 \mathrm{~ms}$ ) voltage-dependent signal with a fractional fluorescence change of 5 per cent. More recently, Ataka \& Pieribone (2002) have developed a similar construct, SPARC, with very rapid kinetics. Unfortunately, no signals have been detected in mammalian cells with either of these FP-voltage sensors. They appear to be retained in the endoplasmic reticulum and are only minimally expressed in the extracellular membrane (Baker et al. 2007). Thomas Knopfel and collaborators (Dimitrov et al. 2007) investigated a new class of voltage-sensitive proteins, the voltage-sensitive phosphatase from Ciona intestinalis (Murata et al. 2005). They replaced the phosphatase domain with a FRET pair and this protein, VSFP2.1, both trafficked well to the external membrane of hippocampal neurons and had a voltage-dependent response. VFSP2.1 was faster than FlaSh but slower than SPARC. Recently, Tsutsui et al. (2008) reported a modified version of VSFP2.1, called Mermaid, with a much larger $(20-30 \%)$ signal. Future efforts will be needed to improve the response time of Mermaid and to test the construct in transgenic systems.

\section{Calcium sensors}

There is a large family of genetically encoded fluorescent $\mathrm{Ca}^{2+}$ indicator proteins. Until recently, all protein calcium sensors employed $\mathrm{Ca}^{2+}$ binding to calmodulin $(\mathrm{CaM})$ and the $\mathrm{Ca}^{2+}$-dependent interaction of calmodulin and the CaM binding peptide M13 as a calcium sensor. The CaM-M13 complex was then attached to green fluorescent protein or one of its variants to generate a single-wavelength $\mathrm{Ca}^{2+}$ indicator (Baird et al. 1999; Nagai et al. 2001; Nakai et al. 2001; Zhang et al. 2002; Ohkura et al. 2005; Reiff et al. 2005). Alternatively, the CaM-M13 complex was sandwiched between CFP (cyan fluorescent protein) and YFP (yellow fluorescent protein) to 
generate ratiometric $\mathrm{Ca}^{2+}$ sensors like the 'cameleons' (Miyawaki et al. 1997; Miyawaki 2005). Calmodulinbased calcium sensors function well in invertebrates and lower vertebrates (for review see refs in Miyawaki 2005), but show rather poor performance in mammals (Hasan et al. 2004; Nagai et al. 2004; Diez-Garcia et al. 2005; Garaschuk et al. 2007). Significant improvement in mammalian neurons came from the use of the alternative $\mathrm{Ca}^{2+}$-binding protein Troponin C (TnC; Heim \& Griesbeck 2004; Mank et al. 2006; Heim et al. 2007). The members of the $\mathrm{TnC}$ family have relatively high dynamic range (fourfold increase in signal strength upon changing $\mathrm{Ca}^{2+}$ concentration from 0 to $10 \mathrm{mM}$ ) and rather fast rise and decay times (Garaschuk et al. 2007), and they respond linearly to an increase in $\mathrm{Ca}^{2+}$ concentration within the physiological activity range (Reiff et al. 2005; Heim et al. 2007). TnC-based indicators can be expressed transgenically in mice and allow in vivo imaging of neural function with single-cell and even subcellular resolution (Heim et al. 2007). While recent developments significantly improved the properties of protein calcium sensors, when compared with organic $\mathrm{Ca}^{2+}$ indicators the protein sensors are still inferior when $\mathrm{Ca}^{2+}$ sensitivity and/or dynamic range of the indicator are considered (see table 1 in Garaschuk et al. 2007).

Over the past 20 years, there have been remarkable (and unanticipated) advances in the kinds of measurements that are possible from mammalian central nervous systems. Membrane potential can be recorded from dendrites and spines; calcium signals can be recorded from hundreds of neurons simultaneously. Optical recordings already provide unique insights into brain activity and organization. Increases in measurement sensitivity are likely because of efforts made to improve the dyes and increase the efficiency of two-photon microscopes. Improvements in genetically encoded sensors will make it possible to record the activity of individual neuron types in the brainstem. Thus, it seems possible that these methods will become even more powerful tools for studying neuronal origins of respiratory activity.

The authors are indebted to their collaborators Vicencio Davila, Amiram Grinvald, Kohtaro Kamino, Les Loew, Bill Ross, Brian Salzberg, Dejan Vucinic, Matt Wachowiak, Alan Waggoner and Jian-young Wu for numerous discussions about optical methods. The experiments carried out in our laboratories were supported by NIH grants DC05259 and NS42739, Deutsche Forschungsgemeinschaft (SFB 391 and SFB 596) and the Bundesministerium für Bildung und Forschung (NGFN-2).

\section{REFERENCES}

Antic, S. 2003 Action potentials in basal and oblique dendrites of rat neocortical pyramidal neurons. F. Physiol. 550, 35-50. (doi:10.1113/jphysiol.2002.033746)

Antic, S. \& Zecevic, D. 1995 Optical signals from neurons with internally applied voltage-sensitive dyes. f. Neurosci. 15, 1392-1405.

Antic, S., Major, G. \& Zecevic, D. 1999 Fast optical recording of membrane potential changes from dendrites of pyramidal neurons. F. Neurophysiol. 82, 1615-1621.

Antic, S., Wuskell, J. P., Loew, L. \& Zecevic, D. 2000 Functional profile of the giant metacerebral neuron of Helix aspersa: temporal and spatial dynamics of electrical activity in situ. F. Physiol. 527, 55-69. (doi:10.1111/ j.1469-7793.2000.00055.x)

Ataka, K. \& Pieribone, V. A. 2002 A genetically-targetable fluorescent probe of channel gating with rapid kinetics. Biophys. F. 82, 509-516. (doi:10.1016/S0006-3495 (02)75415-5)

Baird, G. S., Zacharias, D. A. \& Tsien, R. Y. 1999 Circular permutation and receptor insertion within green fluorescent proteins. Proc. Natl Acad. Sci. USA 96, 11 241-11 246. (doi:10.1073/pnas.96.20.11241)

Baker, B. J., Lee, H., Pieribone, V. A., Cohen, L. B., Isacoff, E. Y., Knopfel, T. \& Kosmidis, E. K. 2007 Fluorescent protein voltage sensors exhibit low plasma membrane expression in mammalian cells. F. Neurosci. Methods 161, 32-38. (doi:10.1016/j.jneumeth.2006.10.005)

Berridge, M. J., Lipp, P. \& Bootman, M. D. 2000 The versatility and universality of calcium signaling. Nat. Rev. Mol. Cell. Biol. 1, 11-21. (doi:10.1038/35036035)

Blasdel, G. G. \& Salama, G. 1986 Voltage-sensitive dyes reveal a modular organization in monkey striate cortex. Nature 321, 579-585. (doi:10.1038/321579a0)

Bonhoeffer, T. \& Grinvald, A. 1993 The layout of isoorientation domains in area 18 of cat visual cortex: optical imaging reveals a pinwheel-like organization. F. Neurosci. 13, 4157-4180.

Bouevitch, O., Lewis, A., Pinevsky, I., Wuskell, J. \& Loew, L. 1993 Probing membrane potential with nonlinear optics. Biophys. f. 65, 672-679. (doi:10.1016/S0006-3495 (93) 81126-3)

Braddick, H. J. J. 1960 Photoelectric photometry. Rep. Prog. Phys. 23, 154-175. (doi:10.1088/0034-4885/23/1/303)

Briggman, K. L. \& Kristan, W. B. 2006 Imaging dedicated and multifunctional neural circuits generating distinct behaviors. F. Neurosci. 26, 10 925-10 933. (doi:10.1523/ JNEUROSCI.3265-06.2006)

Brustein, E., Marandi, N., Kovalchuk, Y., Drapeau, P. \& Konnerth, A. 2003 In vivo monitoring of neuronal network activity in zebrafish by two-photon $\mathrm{Ca}^{2+}$ imaging. Pflugers Arch. 446, 766-773. (doi:10.1007/s00424-003-1138-4)

Cacciatore, T. W., Brodfuehrer, P. D., Gonzalez, J. E., Jiang, T., Adams, S. R., Tsien, R. Y., Kristan Jr, W. B. \& Kleinfeld, D. 1999 Identification of neural circuits by imaging coherent electrical activity with FRET-based dyes. Neuron 23, 449-459. (doi:10.1016/S0896-6273(00)80799-0)

Canepari, M., Djurisic, M. \& Zecevic, D. 2007 Dendritic signals from rat hippocampal CA1 pyramidal neurons during coincident pre- and post-synaptic activity: a combined voltage- and calcium-imaging study. F. Physiol. 580, 463-484. (doi:10.1113/jphysiol.2006.125005)

Cohen, L. B. \& Keynes, R. D. 1971 Changes in light scattering associated with the action potential in crab nerves. f. Physiol. 212, 259-275.

Cohen, L. B. \& Lesher, S. 1986 Optical monitoring of membrane potential: methods of multisite optical measurement. Soc. Gen. Physiol. Ser. 40, 71-99.

Cohen, L. B. \& Salzberg, B. M. 1978 Optical measurement of membrane potential. Rev. Physiol. Biochem. Pharmacol. $83,35-88$.

Cohen, L. B., Keynes, R. D. \& Hille, B. 1968 Light scattering and birefringence changes during nerve activity. Nature 218, 438-441. (doi:10.1038/218438a0)

Davila, H. V., Salzberg, B. M., Cohen, L. B. \& Waggoner, A. S. 1973 A large change in axon fluorescence that provides a promising method for measuring membrane potential. Nat. New Biol. 241, 159-160.

Diez-Garcia, J., Matsushita, S., Mutoh, H., Nakai, J., Ohkura, M., Yokoyama, J., Dimitrov, D. \& Knopfel, T. 2005 Activation of cerebellar parallel fibers monitored in transgenic mice expressing a fluorescent $\mathrm{Ca}^{2+}$ indicator 
protein. Eur. F. Neurosci. 22, 627-635. (doi:10.1111/j. 1460-9568.2005.04250.x)

Dimitrov, D., He, Y., Mutoh, H., Baker, B. J., Cohen, L., Akemann, W. \& Knopfel, T. 2007 Engineering and characterization of an enhanced fluorescent protein voltage sensor. PLoS ONE 2, e440. (doi:10.1371/journal. pone.0000440)

Djurisic, M., Antic, S., Chen, W.-R. \& Zecevic, D. 2004 Voltage imaging from dendrites of mitral cells: EPSP attenuation and spike trigger zones. $\mathcal{F}$. Neurosci. 24, 6703-6714. (doi:10.1523/JNEUROSCI.0307-04.2004)

Djurisic, M., Popovic, M., Carnevale, N. \& Zecevic, D. 2008 Functional structure of the mitral cell dendritic tuft in the rat olfactory bulb. F. Neurosci. 28, 4057-4068. (doi:10. 1523/JNEUROSCI.5296-07.2008)

Dombeck, D. A., Sacconi, L., Blanchard-Desce, M. \& Webb, W. W. 2005 Optical recording of fast neuronal membrane potential transients in acute mammalian brain slices by second-harmonic generation microscopy. F. Neurophysiol. 94, 3628-3636. (doi:10.1152/jn.00416.2005)

Friedrich, R. W. \& Korsching, S. I. 1997 Combinatorial, and chemotropic odorant coding in the zebrafish olfactory bulb visualized by optical imaging. Neuron 18, 737-752. (doi:10.1016/S0896-6273(00)80314-1)

Fromherz, P., Dambacher, K. H., Ephardt, H., Lambacher, A., Muller, C. O., Neigl, R., Schaden, H., Schenk, O. \& Vetter, T. 1991 Fluorescent dyes as probes of voltage transients in neuron membranes: progress report. Ber. Bunsenges. Phys. Chem. 95, 1333-1345.

Garaschuk, O., Milos, R. I. \& Konnerth, A. 2006 Targeted bulk-loading of fluorescent indicators for two-photon brain imaging in vivo. Nat. Prot. 1, 380-386. (doi:10. 1038/nprot.2006.58)

Garaschuk, O., Griesbeck, O. \& Konnerth, A. 2007 Troponin C-based biosensors: a new family of genetically encoded indicators for in vivo calcium imaging in the nervous system. Cell Calcium 42, 351-361. (doi:10. 1016/j.ceca.2007.02.011)

Gonzalez, J. E. \& Tsien, R. Y. 1995 Voltage sensing by fluorescence energy transfer in single cells. Biophys. F. 69, 1272-1280. (doi:10.1016/S0006-3495(95)80029-9)

Grinvald, A., Hildesheim, R., Farber, I. C. \& Anglister, L. 1982 Improved fluorescent probes for the measurement of rapid changes in membrane potential. Biophys. f. 39, 301-308. (doi:10.1016/S0006-3495(82)84520-7)

Grinvald, A., Lieke, E., Frostig, R. D., Gilbert, C. D. \& Wiesel, T. N. 1986 Functional architecture of cortex revealed by optical imaging of intrinsic signals. Nature 324, 361-364. (doi:10.1038/324361a0)

Grinvald, A., Frostig, R. D., Lieke, E. \& Hildesheim, R. 1988 Optical imaging of neuronal activity. Physiol. Rev. 68, 1285-1366.

Gross, E., Bedlack, R. S. \& Loew, L. M. 1994 Dualwavelength ratiometric fluorescence measurements of the membrane dipole potential. Biophys. f. 67, 208-216. (doi:10.1016/S0006-3495(94)80471-0)

Grynkiewicz, G., Poenie, M. \& Tsien, R. Y. 1985 A new generation of $\mathrm{Ca}^{2+}$ indicators with greatly improved fluorescence properties. F. Biol. Chem. 260, 3440-3450.

Gupta, R. K., Salzberg, B. M., Grinvald, A., Cohen, L. B., Kamino, K., Lesher, S., Boyle, M. B., Waggoner, A. S. \& Wang, C. H. 1981 Improvements in optical methods for measuring rapid changes in membrane potential. 7. Membr. Biol. 58, 123-137. (doi:10.1007/BF01870975)

Hamer, F. M. 1964 The cyanine dyes and related compounds. New York, NY: Wiley.

Hasan, M. T. et al. 2004 Functional fluorescent $\mathrm{Ca}^{2+}$ indicator proteins in transgenic mice under TET control. PLoS Biol. 2, 763-775. (doi:10.1371/journal.pbio.0020163)
Heim, N. \& Griesbeck, O. 2004 Genetically encoded indicators of cellular calcium dynamics based on troponin $\mathrm{C}$ and green fluorescent protein. F. Biol. Chem. 279, 14 280-14 286. (doi:10.1074/jbc.M312751200)

Heim, N., Garaschuk, O., Friedrich, M. W., Mank, M., Milos, R. I., Kovalchuk, Y., Konnerth, A. \& Griesbeck, O. 2007 Improved calcium imaging in transgenic mice expressing a troponin-C based biosensor. Nat. Methods 4, 127-129. (doi:10.1038/nmeth1009)

Helmchen, F. \& Denk, W. 2005 Deep tissue two-photon microscopy. Nat. Methods 2, 932-940. (doi:10.1038/ nmeth818)

Helmchen, F., Imoto, K. \& Sakmann, B. $1996 \mathrm{Ca}^{2+}$ buffering and action potential-evoked $\mathrm{Ca}^{2+}$ signaling in dendrites of pyramidal neurons. Biophys. f. 70, 1069-1081. (doi:10.1016/S0006-3495(96)79653-4)

Homma, R., Baker, B., Jin, L., Garaschuk, O., Konnerth, A., Cohen, L. B., Bleau, C. X. \& Zecevic, D. 2009 Wide-field and two-photon imaging of brain activity with voltage- and calcium-sensitive dyes. In Dynamic imaging: towards quantitative understanding of brain function. (ed. F. Hyder). Methods Mol. Biol. 489, pp. 43-79.

Inoue, S. 1986 Video microscopy, p. 128. New York, NY: Plenum Press.

Kerr, J. N., Greenberg, D. \& Helmchen, F. 2005 Imaging input and output of neocortical networks in vivo. Proc. Natl Acad. Sci. USA 102, $14063-14$ 068. (doi:10.1073/ pnas.0506029102)

Kleinfeld, D. \& Delaney, K. R. 1996 Distributed representation of vibrissa movement in the upper layers of somatosensory cortex revealed with voltage-sensitive dyes. F. Comp. Neurol. 375, 89-108. (doi:10.1002/(SICI)1096-9861 (19961104)375:1<89::AID-CNE6>3.0.CO;2-K)

Li, J., Mack, J. A., Souren, M., Yaksi, E., Higashijima, S., Mione, M., Fetcho, J. R. \& Friedrich, R. W. 2005 Early development of functional spatial maps in the zebrafish olfactory bulb. F. Neurosci. 25, 5784-5795. (doi:10. 1523/JNEUROSCI.0922-05.2005)

Loew, L. M. 1993 Confocal microscopy of potentiometric fluorescent dyes. Methods Cell Biol. 38, 195-209. (doi:10.1016/S0091-679X(08)61003-1)

Loew, L. M., Cohen, L. B., Salzberg, B. M., Obaid, A. L. \& Bezanilla, F. 1985 Charge-shift probes of membrane potential. Characterization of aminostyrylpyridinium dyes on the squid giant axon. Biophys. F. 47, 71-77. (doi:10.1016/S0006-3495(85)83878-9)

Loew, L. M., Cohen, L. B., Dix, J., Fluhler, E. N., Montana, V., Salama, G. \& Wu, J. Y. 1992 A napthyl analog of the aminostyryl pyridinium class of potentiometric membrane dyes shows consistent sensitivity in a variety of tissue, cell, and model membrane preparations. f. Membr. Biol. 130, 1-10. (doi:10.1007/BF00233734)

Malmstadt, H. V., Enke, C. G., Crouch, S. R. \& Harlick, G. 1974 Electronic measurements for scientists. Menlo Park, CA: Benjamin.

Mank, M., Reiff, D. F., Heim, N., Friedrich, M. W., Borst, A. \& Griesbeck, O. 2006 A FRET-based calcium biosensor with fast signal kinetics and high fluorescence change. Biophys. F. 90, 1790-1796. (doi:10.1529/biophysj.105. 073536)

Millard, A. C., Jin, L., Wuskell, J. P., Boudreau, D. M., Lewis, A. \& Loew, L. M. 2005 Wavelength- and timedependence of potentiometric nonlinear optical signals from styryl dyes. F. Membr. Biol. 208, 103-111. (doi:10. 1007/s00232-005-0823-y)

Miyawaki, A. 2005 Innovations in the imaging of brain functions using fluorescent proteins. Neuron 48, 189-199. (doi:10.1016/j.neuron.2005.10.003)

Miyawaki, A., Llopis, J., Heim, R., McCaffery, J. M., Adams, J. A., Ikura, M. \& Tsien, R. Y. 1997 Fluorescent indicators 
for $\mathrm{Ca}^{2+}$ based on green fluorescent proteins and calmodulin. Nature 388, 882-887. (doi:10.1038/42264)

Momose-Sato, Y., Sato, K., Sakai, T., Hirota, A., Matsutani, K. \& Kamino, K. 1995 Evaluation of optimal voltagesensitive dyes for optical measurement of embryonic neural activity. F. Membr. Biol. 144, 167-176. (doi:10. 1007/BF00232802)

Murata, Y., Iwasaki, H., Sasaki, M., Inaba, K. \& Okamura, Y. 2005 Phosphoinositide phosphatase activity coupled to an intrinsic voltage sensor. Nature 435, 1239-1243. (doi:10.1038/nature03650)

Nagai, T., Sawano, A., Park, E. S. \& Miyawaki, A. 2001 Circularly permuted green fluorescent proteins engineered to sense $\mathrm{Ca}^{2+}$. Proc. Natl Acad. Sci. USA 98, 3197-3202. (doi:10.1073/pnas.051636098)

Nagai, T., Yamada, S., Tominaga, T., Ichikawa, M. \& Miyawaki, A. 2004 Expanded dynamic range of fluorescent indicators for $\mathrm{Ca}^{2+}$ by circularly permuted yellow fluorescent proteins. Proc. Natl Acad. Sci. USA 101, 10 554-10 559. (doi:10.1073/pnas.0400417101)

Nakai, J., Ohkura, M. \& Imoto, K. 2001 A high signal-to-noise $\mathrm{Ca}^{2+}$ probe composed of a single green fluorescent protein. Nat. Biotechnol. 19, 137-141. (doi:10.1038/84397)

Nakashima, M., Yamada, S., Shiono, S., Maeda, M. \& Satoh, F. 1992 448-detector optical recording system: development and application to Aplysia gill-withdrawal reflex. IEEE Trans. Biomed. Eng. 39, 26-36. (doi:10. $1109 / 10.108124)$

Neher, E. \& Augustine, G. J. 1992 Calcium gradients and buffers in bovine chromaffin cells. F. Physiol. 450, $273-301$.

Niell, C. M. \& Smith, S. J. 2005 Functional imaging reveals rapid development of visual response properties in the zebrafish tectum. Neuron 45, 941-951. (doi:10.1016/j. neuron.2005.01.047)

Nimmerjahn, A., Kirchhoff, F., Kerr, J. N. D. \& Helmchen, F. 2004 Sulforhodamine 101 as a specific marker of astroglia in the neocortex in vivo. Nat. Methods 1, 31-37. (doi:10.1038/nmeth706)

Ohki, K., Chung, S., Ch'ng, Y. H., Kara, P. \& Reid, R. C. 2005 Functional imaging with cellular resolution reveals precise micro-architecture in visual cortex. Nature 433, 597-603. (doi:10.1038/nature03274)

Ohkura, M., Matsuzaki, M., Kasai, H., Imoto, K. \& Nakai, J. 2005 Genetically encoded bright $\mathrm{Ca}^{2+}$ probe applicable for dynamic $\mathrm{Ca}^{2+}$ imaging of dendritic spines. Anal. Chem. 77, 5861-5869. (doi:10.1021/ac0506837)

Onimaru, H. \& Homma, I. 2005 Optical imaging of respiratory neuron activity from the dorsal view of the lower brainstem. Clin. Exp. Pharmacol. Physiol. 32, 297-301. (doi:10.1111/j.1440-1681.2005.04187.x)

Orbach, H. S., Cohen, L. B. \& Grinvald, A. 1985 Optical mapping of electrical activity in rat somatosensory and visual cortex. F. Neurosci. 5, 1886-1895.

Palmer, L. M. \& Stuart, G. J. 2006 Site of action potential initiation in layer 5 pyramidal neurons. F. Neurosci. 26, 1854-1863. (doi:10.1523/JNEUROSCI.4812-05.2006)

Reiff, D. F., Ihring, A., Guerrero, G., Isacoff, E. Y., Joesch, M., Nakai, J. \& Borst, A. 2005 In vivo performance of genetically encoded indicators of neural activity in flies. f. Neurosci. 25, 4766-4778. (doi:10.1523/JNEUROSCI. 4900-04.2005)

Rohr, S. \& Salzberg, B. M. 1994 Multiple site optical recording of transmembrane voltage in patterned growth heart cell cultures: assessing electrical behavior, with microsecond resolution, on a cellular and subcellular scale. Biophys. F. 67, 1301-1315. (doi:10.1016/S00063495(94)80602-2)

Ross, W. N., Salzberg, B. M., Cohen, L. B. \& Davila, H. V. 1974 A large change in dye absorption during the action potential. Biophys. f. 14, 983-986. (doi:10.1016/S00063495(74)85963-1)

Salzberg, B. M., Grinvald, A., Cohen, L. B., Davila, H. V. \& Ross, W. N. 1977 Optical recording of neuronal activity in an invertebrate central nervous system: simultaneous monitoring of several neurons. F. Neurophysiol. 40, $1281-1291$.

Siegel, M. S. \& Isacoff, E. Y. 1997 A genetically encoded optical probe of membrane voltage. Neuron 19, 735-741. (doi:10.1016/S0896-6273(00)80955-1)

Sjostrom, P. J., Rancz, E. A., Roth, A. \& Hausser, M. 2008 Dendritic excitability and synaptic plasticity. Physiol. Rev. 88, 769-840. (doi:10.1152/physrev.00016.2007)

Stosiek, C., Garaschuk, O., Holthoff, K. \& Konnerth, A. 2003 In vivo two-photon calcium imaging of neuronal networks. Proc. Natl Acad. Sci. USA 100, 7319-7324. (doi:10.1073/pnas.1232232100)

Stuart, G. J. \& Sakmann, B. 1994 Active propagation of somatic action potentials into neocortical pyramidal cell dendrites. Nature 367, 69-72. (doi:10.1038/367069a0)

Sullivan, M. R., Nimmerjahn, A., Sarkisov, D. V., Helmchen, F. \& Wang, S. S. 2005 In vivo calcium imaging of circuit activity in cerebellar cortex. F. Neurophysiol. 94, 1636-1644. (doi:10.1152/jn.01013.2004)

Teisseyre, T. Z., Millard, A. C., Yan, P., Wuskell, J. P., Wei, M. D., Lewis, A. \& Loew, L. M. 2007 Nonlinear optical potentiometric dyes optimized for imaging with 1064-nm light. F. Biomed. Opt. 12, 044001. (doi:10.1117/1. 2772276)

Tsau, Y., Wenner, P., O’Donovan, M. J., Cohen, L. B., Loew, L. M. \& Wuskell, J. P. 1996 Dye screening and signal-to-noise ratio for retrogradely transported voltagesensitive dyes. F. Neurosci. Methods 70, 121-129. (doi:10.1016/S0165-0270(96)00109-4)

Tsien, R. Y. 1981 A non-disruptive technique for loading calcium buffers and indicators into cells. Nature 290, 527-528. (doi:10.1038/290527a0)

Tsien, R. W. \& Tsien, R. Y. 1990 Calcium channels, stores, and oscillations. Annu. Rev. Cell Biol. 6, 715-760. (doi:10.1146/annurev.cb.06.110190.003435)

Tsutsui, H., Karasawa, S., Okamura, Y. \& Miyawaki, A. 2008 Improving membrane voltage measurements using FRET with new fluorescent proteins. Nat. Methods 8, 683-685. (doi:0.1038/nmeth.1235)

Verkhratsky, A. 2005 Physiology and pathophysiology of the calcium store in the endoplasmic reticulum of neurons. Physiol. Rev. 85, 201-279. (doi:10.1152/physrev.00004. 2004)

Wachowiak, M. \& Cohen, L. B. 2001 Representation of odorants by receptor neuron input to the mouse olfactory bulb. Neuron 32, 723-735. (doi:10.1016/S08966273(01)00506-2)

Wachowiak, M. \& Cohen, L. B. 2003 Correspondence between odorant-evoked patterns of receptor neuron input and intrinsic optical signals in the mouse olfactory bulb. F. Neurophysiol. 89, 1623-1639. (doi:10.1152/jn. 00747.2002)

Waggoner, A. S. \& Grinvald, A. 1977 Mechanisms of rapid optical changes of potential-sensitive dyes. Ann. NY Acad. Sci. 303, 217-241.

Zecevic, D. 1996 Multiple spike-initiation zones in single neurons revealed by voltage-sensitive dyes. Nature 381, 322-325. (doi:10.1038/381322a0)

Zhang, J., Campbell, R. E., Ting, A. Y. \& Tsien, R. Y. 2002 Creating new fluorescent probes for cell biology. Nat. Rev. Mol. Cell Biol. 3, 906-918. (doi:10.1038/nrm976)

Zochowski, M., Wachowiak, D. M., Falk, C. X., Cohen, L. B., Lam, Y.-W., Antic, S. \& Zecevic, D. 2000 Imaging membrane potential with voltage-sensitive dyes. Biol. Bull. 198, 1-21. (doi:10.2307/1542798) 